\title{
Transport in disordered systems: The single big jump approach
}

\author{
Wanli Wang $\odot,{ }^{1,2, *}$ Alessandro Vezzani,${ }^{3}$ Raffaella Burioni, ${ }^{3}$ and Eli Barkai ${ }^{1,2}$ \\ ${ }^{1}$ Department of Physics, Bar-Ilan University, Ramat-Gan 52900, Israel \\ ${ }^{2}$ Institute of Nanotechnology and Advanced Materials, Bar-Ilan University, Ramat-Gan 52900, Israel \\ ${ }^{3}$ INFN, Gruppo Collegato di Parma, via G.P. Usberti 7/A, 43124 Parma, Italy
}

(Received 18 June 2019; revised manuscript received 31 October 2019; published 12 December 2019)

\begin{abstract}
In a growing number of strongly disordered and dense systems, the dynamics of a particle pulled by an external force field exhibits superdiffusion. In the context of glass-forming systems, supercooled glasses, and contamination spreading in porous media, it was suggested that this behavior be modeled with a biased continuous-time random walk. Here we analyze the plume of particles lagging far behind the mean, with the single big jump principle. Revealing the mechanism of the anomaly, we show how a single trapping time, the largest one, is responsible for the rare fluctuations in the system. These nontypical fluctuations still control the behavior of the mean square displacement, which is the most basic quantifier of the dynamics in many experimental setups. We show how the initial conditions, describing either the stationary state or nonequilibrium case, persist forever in the sense that the rare fluctuations are sensitive to the initial preparation. To describe the fluctuations of the largest trapping time, we modify Fréchet's law from extreme value statistics, taking into consideration the fact that the large fluctuations are very different from those observed for independent and identically distributed random variables.
\end{abstract}

DOI: 10.1103/PhysRevResearch.1.033172

\section{INTRODUCTION}

Diffusion and transport in a vast number of weakly disordered systems follow Gaussian statistics. As a consequence, the packet of the spreading particles is symmetrically spread with respect to the mean $\langle x(t)\rangle$. In contrast, for strongly disordered systems, the packet is found to be non-Gaussian and nonsymmetric [1,2]. Starting on $x=0$, the slowest particles are trapped by the disorder, resulting in a plume of particles lagging far behind the mean $\langle x(t)\rangle$, i.e., the fluctuations are large and break symmetry (see Figs. 1 and 2). Deep energetic and entropic traps, which hinder the motion, are expected to lead to a slowdown of the diffusion. The most frequently used quantifier of diffusion processes is clearly the mean square displacement (MSD). However, in the presence of deep traps, the MSD exhibits superdiffusion. This is not an indication for a fast process, instead it is due to the very slow particles far lagging behind the mean, which lead to very large fluctuations of displacements. Thus slow dynamics of a minority of particles leads to enhanced fluctuations and symmetry breaking with respect to $\langle x(t)\rangle$. Such processes are widespread; in particular, many works focused on the surprising discovery of the superdiffusion in dense environments [3-8]. This was originally investigated in the context of diffusion in disordered material [1-3,9-12], contamination spreading in porous media [13-16], simulation of biased particles in glass-forming

\footnotetext{
*wangw112@1zu.edu.cn

Published by the American Physical Society under the terms of the Creative Commons Attribution 4.0 International license. Further distribution of this work must maintain attribution to the author(s) and the published article's title, journal citation, and DOI.
}

systems [4], and supercooled liquids [6], pulled by a constant force.

Here we investigate the spreading of the packet of particles, using the biased continuous-time random walk (CTRW) [17-19]. Our goal is to characterize precisely the mechanism leading to the large fluctuations. We promote the idea of the single big jump principle. This means that one and only one trapping time is responsible for the rare fluctuations. Thus, in this work we show the relation between the theory of extreme value statistics and the anomalous transport. For that we need to modify the well-known Fréchet law $[20,21]$ which describes extreme events for uncorrelated systems. Similarly, we present an analysis of the far tail of the spreading of the packet of particles, showing the deviations from the Lévy statistics describing the bulk statistics. This is done for both nonstationary and equilibrium initial conditions. While the typical fluctuations in our systems are not sensitive to the initial conditions, the rare fluctuations are, and this we believe is a general theme for systems with fat-tailed statistics.

We will relate the position of the random walker $x(t)$ and the longest trapping interval $\tau_{\max }$. The typical fluctuations of both observables were considered previously and were shown to behave as if they were composed of independent and identically distributed events, namely, the Lévy stable law and Fréchet's law hold for typical fluctuations [Eqs. (15) and (31) below]. We show below how these laws must be corrected when dealing with the far tail. In turn, the standard Cramer's theorem from large-deviation theory [22], which identifies the large fluctuations with the accumulation of many small steps, fails in this case studied here. More precisely, we claim below that one can obtain two limiting laws for both $x(t)$ and $\tau_{\max }$; the first is the just-mentioned Lévy and Fréchet laws and the second is an infinite density, i.e., a non-normalized state. 

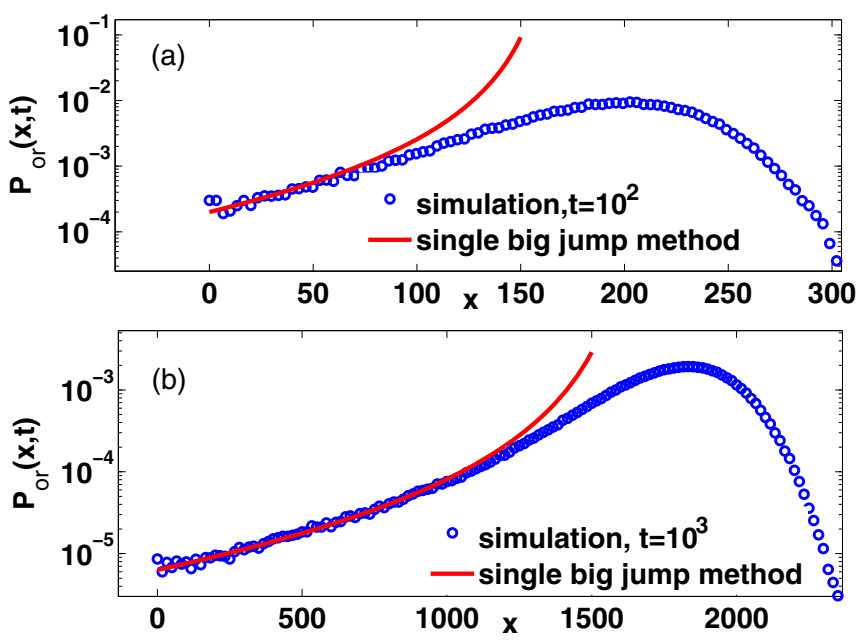

FIG. 1. Density of positions of particles for an ordinary CTRW model. The spreading packet is non-Gaussian. The left plume of particles is due to the long trapping times, which implies that some particles are moving by far slower if compared with the mean $\langle x(t)\rangle$. Somewhat paradoxically, these slow particles lead to superdiffusion as the MSD grows as $t^{3-\alpha}$ [1]. In this work we show how rare events in this process are determined by the largest trapping times. In turn, the rare events control the behavior of the MSD. The typical fluctuations are defined for $x \sim\langle x(t)\rangle$, i.e., close to the peak of the packet, while we focus on the rare fluctuations shown by the red solid line. The parameters are $a=5, \sigma=1$, and $\alpha=1.5$ [see Eqs. (2) and (4)].

What is the principle of big jump? Many works have focus on the dominance of one big jump in a stochastic process. For example, consider the activation process of a particle over a barrier, modeled with an overdamped Langevin equation.
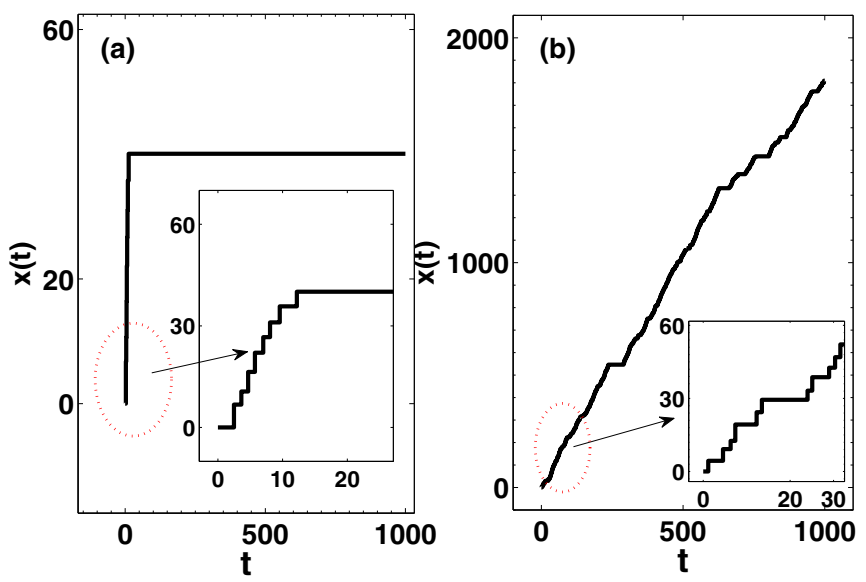

FIG. 2. Two trajectories of particles ending at small and large $x$ when $t=1000$. For the case where $x(t=1000)$ is near the original position, we see a very long waiting time, as the particle is trapped for a time of the order of $t$. In contrast, when $x(t=1000) \simeq\langle x(t)\rangle$, the trapping times are relatively short and comparable to each other. The inset shows the trajectory of the particle at a short time. The parameters are the same as in Fig. 1.
If the noise is noncorrelated and Gaussian, this escape is achieved by many small displacements, accumulating to give the rare escape from the well. On the other hand, if the noise is of the Lévy type, one event giving rise to a large fluctuation dominates the escape [23]. Similar ideas hold for the analysis of random partition functions and were used in the study of the Sinai model [24,25]. In the context of a run-and-tumble model and the combination phenomenon, these insights are well understood [26,27]. Roughly speaking, one can see that the largest summand is of the order of the total sum, a theme which is already known.

To be more specific consider $N$ random variables $\left\{\vartheta_{1}, \vartheta_{2}, \ldots, \vartheta_{N}\right\}$. Let $\vartheta_{\text {max }}$ be the maximum of the set and $S_{N}=\sum_{i=1}^{N} \vartheta_{i}$ is the sum. The dominance effect, found, for example, if the $\vartheta_{i}$ are independent and identically distributed random variables drawn from a fat-tailed distribution, is the claim that $S_{N}$ and $\vartheta_{\max }$ are of the same order [28]. More exactly, $S_{N}$ and $\vartheta_{\max }$ scale with $N$ the same way. A more profound case is when the distribution of $\vartheta_{\max }$ is the same as that of $S_{N}$, except for a trivial constant and in a limit to be specified later. This is what we and others refer to as the principle of big jump. This statement was shown to be valid for subexponential independent and identically distributed variables [28] (see also [29,30]). In the independent and identically distributed case, the statement is valid for any $N$, so the $\operatorname{limit} N \rightarrow \infty$ is not at all required. Here our aim is show how the big jump principle holds for diffusion in disorder systems using the CTRW model. We will modify the principle to discuss the largest trapping time and its relation to the position of the random walker, so the principle discussed below is very different if compared to the original; in particular, we depart from the independent and identically distributed case.

In $[31,32]$ we promoted a rate method to the big jump approach which was used to predict nonanalytical behaviors of the far tail of Lévy walk process and the so-called quenched Lévy-Lorentz gas model. In these works, the very basic approach is different from what we have here [see Eq. (9) below]. Further, the connection to the modified Fréchet law and the difference between stationary and nonequilibrium initial conditions are discussed here.

The organization of the paper is as follows. In Sec. II we outline the single big principle and give the corresponding definitions. Nonequilibrium and equilibrium initial conditions are investigated in Secs. III and IV, respectively. We conclude with a discussion. We also present simulation results confirming the theoretical predications.

\section{SINGLE BIG JUMP PRINCIPLE}

\section{A. Model and definition}

We consider two types of biased CTRWs [17-19,33,34]; the first is initiated at time $t=0$ while the second is an equilibrium process. These two models differ in the first trapping time statistics, but otherwise they are identical. Let $\phi(\tau)$ be the probability density function (PDF) of all the sojourn times while $h(\tau)$ is the PDF of the first one. It should be emphasized that the correct choice of $h(\tau)$ depends on the initial conditions. For the widely investigated nonequilibrium initial condition, we assign $h_{\mathrm{or}}(\tau)=\phi(\tau)$ [35]. This time process is 
sometimes called an ordinary renewal process; hence we use the subscript "or" to denote this type of initial condition. In contrast, in an equilibrium situation we use [36-39]

$$
h_{\mathrm{eq}}(\tau)=\frac{\int_{\tau}^{\infty} \phi(y) d y}{\langle\tau\rangle},
$$

where $\langle\tau\rangle=\int_{0}^{\infty} \tau \phi(\tau) d \tau$ is the mean trapping time. We will soon explain the physical meaning of these processes.

We are interested in the position of the random walker $x(t)$, which starts at $x=0$ when $t=0$. After waiting for time $\tau_{1}$, drawn from $h(\tau)$, the particle makes a spatial jump. The PDF of jump size $\chi$ is Gaussian

$$
f(\chi)=\frac{1}{\sqrt{2 \sigma^{2} \pi}} \exp \left[-\frac{(\chi-a)^{2}}{2 \sigma^{2}}\right],
$$

where $a>0$ is the average size of the jumps. Physically, this is determined by an external constant force field that induces a net drift. From Eq. (2) the Fourier transform of $f(\chi)$ is $\tilde{f}(k)=$ $\exp \left(i k a-\sigma^{2} k^{2} / 2\right)$. This yields

$$
\tilde{f}(k) \sim 1+i k a-\frac{\sigma^{2}+a^{2}}{2} k^{2},
$$

with $k \rightarrow 0$. After the jump, say, to $x_{1}$, the particle will pause for time $\tau_{2}$, whose statistical properties are drawn from $\phi(\tau)$. Then the process is renewed. We consider the widely applicable case where the PDF of trapping times is

$$
\phi(\tau)= \begin{cases}0, & \tau<\tau_{0} \\ \alpha \frac{\tau_{0}^{\alpha}}{\tau^{1+\alpha}}, & \tau \geqslant \tau_{0},\end{cases}
$$

with $1<\alpha<2$. As is well known, such a fat-tailed distribution yields a wide range of anomalous behaviors. (See [10,17] for a review on the CTRW and further discussion on physical systems below.) From the Abelian theorem, the Laplace $\tau \rightarrow$ $s$ transform of $\phi(\tau)$ is

$$
\hat{\phi}(s) \sim 1-\langle\tau\rangle s+b_{\alpha} s^{\alpha},
$$

with $b_{\alpha}=\left(\tau_{0}\right)^{\alpha}|\Gamma(1-\alpha)|$ and $s \rightarrow 0$. The leading term is the normalization condition. We focus on $1<\alpha<2$, where the mean $\langle\tau\rangle$ of the waiting time is finite, but not the variance. The term $s^{\alpha}$ comes from the long tail of the waiting times (and it is responsible for the deviations from the normal behavior). Specific values of $\alpha$ for a range of physical systems and models are given in $[10,40]$.

For an equilibrium initial condition the rate of performing a jump is stationary in the sense that for any time $t$ the average number of jumps is

$$
\langle N(t)\rangle=\frac{t}{\langle\tau\rangle},
$$

so the effective rate $1 /\langle\tau\rangle$ is a constant. In contrast, for the ordinary renewal process we have in the long-time limit $\langle N(t)\rangle \sim t /\langle\tau\rangle$; hence for short times the two processes are not identical. Since the mean $\langle\tau\rangle$ is finite, one would expect naively that statistical laws for the two processes will be identical in the long-time limit. While this is correct for some observables, for others this is false. The prominent example is the MSD. In particular, for the calculation of the rare events one must make the distinction between the two models (discussed below). An equilibrium initial condition
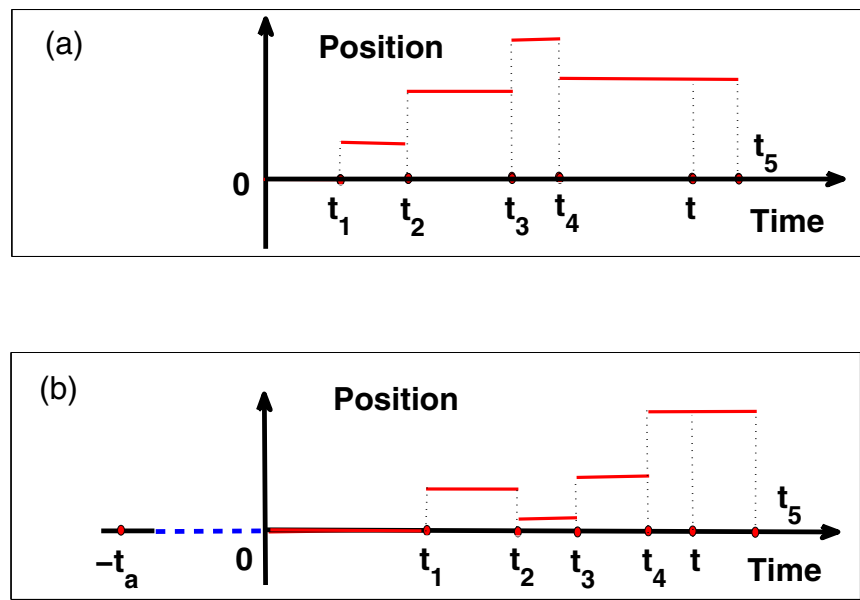

FIG. 3. Illustrations of (a) an ordinary CTRW when the process begins at time $t=0$ and $x=0$ and (b) an equilibrium CTRW. (b) describes an ongoing equilibrium process, i.e., a stationary case where the dynamics started long before the start of the observation (see the blue dashed line for an illustration). The $t_{i}$ corresponds to the time when the $i$ th event occurs, and the backward recurrence time is $B_{t}=t-t_{4}$. The only difference between these two processes is the statistics of the waiting time of the first step. However, due to the disorder, in particular the power-law trapping time distribution, this difference crucially influences the rare events and also the behavior of the MSD.

is found when the particle is inserted in the medium long before the process begins, more specifically, when the process starts at some time $-t_{a}$ before the measurement begins at time $t=0$ and in the limit $t_{a} \rightarrow \infty$. All along we consider the displacement of the particle compared to its initial position, namely, we assign $x(0)=0$. For a schematic presentation of the random processes see Fig. 3 .

Nonequilibrium initial conditions are found when the processes are initiated at time $t=0$. For example, in ScherMontroll theory [41], a flash of light excites charge carriers at time $t=0$ and then the process of diffusion begins; then we have an ordinary process. Mathematically, these two models merely differ by the statistics of the waiting time of the first step, and hence it is interesting to compare them, to see whether or not this seemingly small modification of the model is important in the long-time limit. For a Poisson process the two models are identical. In contrast, for the heavy-tailed processes under investigation, we find from Eqs. (1) and (4) that

$$
h_{\mathrm{eq}}(\tau) \sim \frac{\left(\tau_{0}\right)^{\alpha}}{\langle\tau\rangle} \tau^{-\alpha} .
$$

As $1<\alpha<2$ we see that the average time for the first waiting time diverges (but not for the second, etc.). This means that in a stationary state the process is slower if compared to the ordinary case; hence we expect that in this case particles will be lagging even farther behind the mean displacement.

Let us discuss the applicability of the CTRW model. As mentioned, Scher and Montroll showed how this theory describes the diffusion of charge carriers in disordered media. In some experiments, one can find $\alpha=T / T_{g}$, where $T$ is the temperature and $T_{g}$ is the measure of the disorder. This is 
also the case for the Bouchaud trap model describing glassy dynamics [10]. In the context of contamination spreading, a biased CTRW is used with $\alpha=1.73$ [16]. Based on numerical simulations, Winter et al. [4] and Schroer and Heuer [6] showed the superdiffusive behavior and related the dynamics to the biased CTRW. In these systems one expects that at very long times we find normal diffusion. There are also many examples of a CTRW without bias $[17,40,42,43]$. It is interesting to add a bias in these systems to compare the effect discussed here.

\section{B. Main results: The big jump principle}

Transport and diffusion processes, either normal or anomalous, are composed of a large number of displacements. Hence statistical laws, like the central limit theorem, are useful tools describing universal aspects of the phenomenon. In our case a single event is controlling the statistics of the spreading packet at its tail. Let $\left\{\tau_{1}, \tau_{2}, \ldots, \tau_{N}, B_{t}\right\}$ be the set of waiting times between jump events and $\sum_{i=1}^{N} \tau_{i}+B_{t}=t$ is the measurement time. Here $B_{t}$, called the backward recurrence time, is the time elapsing between the moment of last jump $t_{N}=\sum_{i=1}^{N} \tau_{i}$ and the measurement time $t ; N$ is the random number of jumps in $(0, t)$ [44]. We define the largest waiting time according to

$$
\tau_{\max }=\max \left\{\tau_{1}, \tau_{2}, \ldots, \tau_{N}, B_{t}\right\} .
$$

One main conclusion of this paper is that the statistics of $\tau_{\max }$ determine the fluctuations of the position $x(t)$ of the biased random walker. This holds for rare fluctuations of $x(t)$, which still control the behavior of the most typical observable in the field: the MSD.

Due to the fat-tailed distribution of the trapping time $\phi(\tau)$ and using basic arguments from extreme value statistics of independent and identically distributed random variables, one expects that the typical fluctuations scale as $\tau_{\max } \propto t^{1 / \alpha}$, while for a thin-tailed distribution of waiting time, e.g., $\phi(\tau)=$ $\exp (-\tau)$, we have $\tau_{\max } \propto \ln (t)$ [20]. For the latter example $\propto$ means that $\tau_{\max }$ is of the order of $\ln (t)$ and similarly for the former case. While we are not dealing with independent and identically distributed random variables, the constraint is weak in the sense that it does not modify the typical fluctuations (discussed below and in Refs. [28,45]). Note that all these scalings, i.e., $\tau_{\max } \propto t^{1 / \alpha}$ and $\tau_{\max } \propto \ln (t)$, describe typical fluctuations, sometimes called bulk fluctuations. These fluctuations are described by normalized densities, specified by Fréchet's law and the Gumbel law. In contrast, here we focus on rare fluctuations, that is to say, both $\tau_{\max }$ and $t$ are comparable.

When Eq. (4) holds, for the biased CTRW we will demonstrate that for small $x$, i.e., the left plume in Fig. 1,

$$
x \doteqdot \frac{t-\tau_{\max }}{\langle\tau\rangle} a,
$$

where $\doteqdot$ indicates that the random variables on both sides follow the same distribution. However, the PDFs describing the position of the particle $x$ when $x$ is not small and of $\tau_{\max }$ are far from being identical; they will be calculated below. The meaning of small $x$ and large $\tau_{\max }$ will soon become clear when we formulate the problem more precisely. For
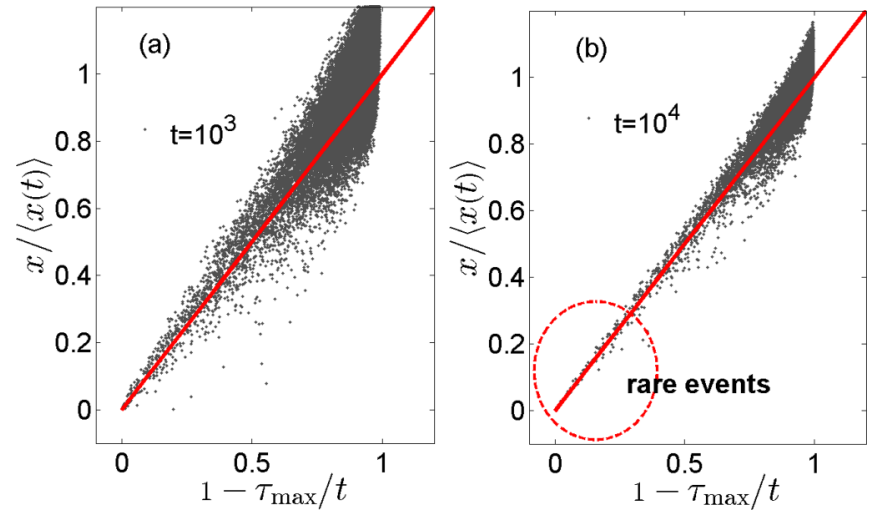

FIG. 4. Correlation plot between $1-\tau_{\max } / t$ and $x /\langle x(t)\rangle$ predicated in Eq. (9) for the biased ordinary CTRW process. Here we choose $a=1, \alpha=1.5, \sigma=\sqrt{2}$, and $\langle\tau\rangle=1$. The dots are simulation results obtained by generating $10^{5}$ trajectories and the red solid line is obtained from Eq. (9) by switching random variables to a dimensionless form, i.e., $x /\langle x(t)\rangle \sim x /(a t /\langle\tau\rangle) \doteqdot\left(1-\tau_{\max } / t\right)$. The evidently strong correlations, circled in (b), indicate that a single trapping event is responsible for the statistics of rare events.

now, based on Figs. 1 and 2, we see that Eq. (9) works well when $x \ll\langle x(t)\rangle$ and $\tau_{\max } \simeq t$. For example, when $x<50 \ll$ $\langle x(t)\rangle \simeq 1667$ in Fig. 1(b) or for the trajectory of Fig. 2(a), where $\tau_{\max }=988$, when $t=1000, x \simeq 40 \ll\langle x(t)\rangle \simeq 1667$. Equation (9) means that the distribution of $x \ll\langle x(t)\rangle$ is the same as the average size of the jumps $a$ times the typical number of jumps made in $\left(0, t-\tau_{\max }\right)$, which is the time "free" of the longest waiting time. A correlation plot based on Eq. (9) is demonstrated numerically in Fig. 4. Using simulations of the ordinary CTRW process, we generate trajectories and search for positions of the random walkers at time $t$ and record $\tau_{\max }$. Then we plot the random entries, observing that for small $x$ there is a perfect correlation as predicated by Eq. (9). Such correlation plots indicate that Eq. (9) is working. We call this the principle of the big jump, and it is valid for both stationary and ordinary processes. Here the big jump means the large trapping time (see further discussion on the term big jump and its origin in Sec. V). Now we will analytically derive Eq. (9) and discuss its consequence. For that we obtain the distribution of $\tau_{\max }$ and then of $x$.

Remark 1. Our main results in this paper are Eqs. (27), (37), (46), and (56), which give explicit formulas for the PDFs of $x$ and $\tau_{\max }$ for the two types of processes under investigation. In [31] we promoted a rate formalism to treat similar problems, e.g., the Lévy walk. Here the focus is on the exact calculation of the statistics of rare events for both $\tau_{\max }$ and $x$ and on the relation between these two random variables, i.e., Eq. (9).

\section{Statistics of $\tau_{\max }$}

Let us proceed to derive the general formulas describing the statistics of the longest waiting times which are valid for both the ordinary and equilibrium renewal processes. The case of an ordinary renewal theory was considered previously by Godrèche et al. in Ref. [45]. They investigated the typical fluctuations of $\tau_{\max }$, and these as explained below exhibit 
behavior identical to a classical case of extreme value statistics, namely, Fréchet's law holds for typical fluctuations. Here our goal is very different; we aim to obtain the rare events, namely, investigate the behavior when $\tau_{\max }$ is of the order $t$. In this case the fluctuations greatly differ from the independent and identically distributed case.

We define the probability that $\tau_{\max }$ is smaller than $L$,

$$
F(t, L)=\operatorname{Prob}\left[\tau_{\max } \leqslant L\right] .
$$

The corresponding PDF is $P_{\tau_{\max }}(t, L)$ and as usual $F(t, L)=$ $\int_{0}^{L} P_{\tau_{\max }}(t, y) d y$. Clearly, the probability depends on the measurement time $t$ and this dependence is especially important for fat-tailed waiting-time PDFs. It is helpful to introduce the joint probability distribution of $\tau_{\max }$ and the number of renewals $N$,

$$
\begin{aligned}
F_{n}(t, L) & =\operatorname{Prob}\left(\tau_{\max } \leqslant L, N=n\right) \\
& =\int_{0}^{L} d \tau_{1} \int_{0}^{L} d \tau_{2} \cdots \int_{0}^{L} d B_{t} P_{\tau_{\max }}\left(\tau_{1}, \tau_{2}, \ldots, \tau_{n}, B_{t}\right)
\end{aligned}
$$

$$
\hat{F}_{n}(s, L)= \begin{cases}\int_{0}^{L} \exp \left(-s \tau_{1}\right) \int_{\tau_{1}}^{\infty} h(\tau) d \tau d \tau_{1}, & n=0 \\ \int_{0}^{L} \exp (-s \tau) h\left(\tau_{1}\right) d \tau_{1}\left(\int_{0}^{L} \exp (-s \tau) \phi(\tau) d \tau\right)^{n-1} \int_{0}^{L} \exp \left(-s B_{t}\right) \int_{B_{t}}^{\infty} \phi(\tau) d \tau d B_{t}, & n \geqslant 1 .\end{cases}
$$

where $h(\cdot)$ in the third line is governed by the process we investigate and $\Phi(t)$ is determined by the type of the process and the number of renewals

$$
\Phi(t)= \begin{cases}\int_{t}^{\infty} h(\tau) d \tau & \text { for } n=0 \text { (equilibrium process) } \\ \int_{t}^{\infty} \phi(\tau) d \tau & \text { otherwise. }\end{cases}
$$

Taking the Laplace transform with respect to $t$, we find
The case $n=0$ corresponds to realizations with no renewals during the time interval $(0, t)$. One can check that $\sum_{n=0}^{\infty} \hat{F}_{n}(s, L \rightarrow \infty)=1 / s$. This means that the density of $\tau_{\max }$ is normalized. The sum of $n$ from zero to infinity gives

$$
\begin{aligned}
\hat{F}(s, L)= & \int_{0}^{L} \exp \left(-s \tau_{1}\right) \int_{\tau_{1}}^{\infty} h(\tau) d \tau d \tau_{1} \\
& +\int_{0}^{L} \exp \left(-s \tau_{1}\right) h\left(\tau_{1}\right) d \tau_{1} \\
& \times \frac{\int_{0}^{L} \exp \left(-s B_{t}\right) \int_{B_{t}}^{\infty} \phi(y) d y d B_{t}}{1-\int_{0}^{L} \exp (-s \tau) \phi(\tau) d \tau} .
\end{aligned}
$$

The first term is related to the survival probability and the second term corresponds to the probability that at least one renewal happened in $(0, t)$. For the equilibrium renewal process, we insert Eq. (1) into Eq. (14), while for the ordinary case we use $h_{\mathrm{or}}(\tau)=\phi(\tau)$. Below, from Eq. (14) we will calculate the far tail of the distribution of $\tau_{\max }$ for the two different processes, i.e., the ordinary process and the equilibrium one, and prove that Eq. (9) is valid for both cases.

\section{ORDINARY PROCESS}

Here we consider the ordinary renewal process and the ordinary CTRW to build the relation between the rare events of positions and the largest waiting times.

\section{A. Rare fluctuations of $\tau_{\max }$}

The aim is to investigate the PDF of $\tau_{\max }$ for the nonequilibrium process which is denoted by $P_{\mathrm{or}, \tau_{\max }}(t, L)$. We first

$$
\begin{aligned}
= & \int_{0}^{L} h\left(\tau_{1}\right) d \tau_{1} \int_{0}^{L} \phi\left(\tau_{2}\right) d \tau_{2} \ldots \\
& \times \int_{0}^{L} \Phi\left(B_{t}\right) d B_{t} \delta\left(t-\left(\sum_{1}^{n} \tau_{j}+B_{t}\right)\right),
\end{aligned}
$$

treat the problem heuristically to calculate the typical fluctuations. Let $\langle N\rangle=t /\langle\tau\rangle$ be the average number of renewals in the long-time limit. For simplification, we neglect $B_{t}$ in Eq. (8) and ignore the constraint $\sum_{i=1}^{N} \tau_{i}+B_{t}=t$; further we replace the random $N$ with $\langle N\rangle$. This means that we treat this problem as if the waiting times are independent and identically distributed random variables, an approximation which turns out to be insufficient in our case, still ignoring the correlation [45]

$$
\begin{aligned}
\operatorname{Prob}\left(\tau_{\max }<L\right) & =\operatorname{Prob}^{N}\left(\tau_{i}<L\right) \\
& \simeq\left[1-\left(\frac{\tau_{0}}{L}\right)^{\alpha}\right]^{N} \\
& \sim \exp \left[-\langle N\rangle\left(\frac{\tau_{0}}{L}\right)^{\alpha}\right] .
\end{aligned}
$$

This is the well-known Fréchet distribution [21]. A closer look reveals a drawback of this treatment of the typical fluctuations, since within this approximation the PDF of $\tau_{\max }$ is $P_{\tau_{\max }}(t, L) \sim \alpha\langle N\rangle\left(\tau_{0}\right)^{\alpha} / L^{1+\alpha}$ for $L \rightarrow \infty$. However, in our setting $\tau_{\max } \leqslant t$. This means that we must modify Fréchet's law at its tail; in other words, the constraint that the sum of all the waiting times and the backward recurrence time is equal to the measurement time $t$ comes into play when $\tau_{\max } \propto t$, as expected. Note that the number of renewals in our case is a random variable (see Fig. 5).

Now we use an exact solution of the problem to calculate the rare events. Considering the nonequilibrium renewal process, we insert $h(\tau)=\phi(\tau)$ into Eq. (13) to get [45]

$$
\int_{L}^{\infty} \hat{P}_{\mathrm{or}, \tau_{\max }}(z) d z=\frac{1}{s} \frac{1}{1+\hat{G}(s, L)},
$$



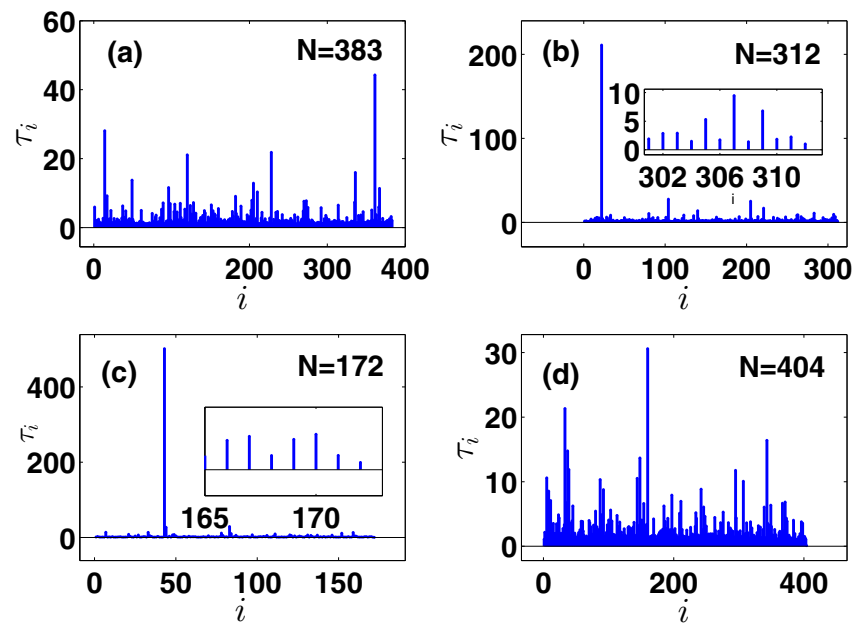

FIG. 5. Random variables $\tau_{i}$ for an ordinary renewal process with Eq. (4) and $\alpha=1.5$. The observation time $t$ is 1000 and $i=$ $100,200, \ldots$ correspond to the 100 th, 200 th, ... waiting times of the fractional renewal process, respectively. Clearly, in our case the number of renewals $N$ is a random variable. Due to the fat-tailed trapping times, the fluctuation of $N$ is large, which come from the large fluctuations of waiting times.

where

$$
\hat{G}(s, L)=\frac{s \exp (s L)}{p_{0}(L)} \int_{0}^{L} p_{0}(t) \exp (-s t) d t,
$$

with the survival probability

$$
p_{0}(t)=\int_{t}^{\infty} \phi(\tau) d \tau \simeq\left(\frac{\tau_{0}}{t}\right)^{\alpha} .
$$

We are interested in the limit $s \rightarrow 0$ (corresponding to long measurement time) and $L \rightarrow \infty$ in such a way that $s L$ remains a constant. As mentioned, the typical fluctuations are described by Fréchet's law (15) and here instead we consider the rare fluctuations. Using Eq. (18), for $L \rightarrow \infty$, Eq. (17) becomes

$$
\hat{G}(s, L) \sim \frac{\exp (s L) s L^{\alpha}\langle\tau\rangle}{\left(\tau_{0}\right)^{\alpha}},
$$

where we have used the limit

$$
\lim _{L \rightarrow \infty} \int_{0}^{L} p_{0}(t) \exp (-s t) d t=\frac{1-\hat{\phi}(s)}{s} \sim\langle\tau\rangle,
$$

with $s \rightarrow 0$. From Eq. (19) we see that $\hat{G}(s, L)$ is large for $s L \rightarrow$ const and $\alpha>1$. According to Eq. (19), we find

$$
\frac{\partial \hat{G}(s, L)}{\partial L} \sim s \hat{G}(s, L)+\frac{\alpha}{L} \hat{G}(s, L)+\cdots .
$$

Note that Eq. (21) can also be derived directly from Eq. (17). Utilizing Eq. (16) and

$$
F(t, L)=\int_{0}^{L} P_{\mathrm{or}, \tau_{\max }}(t, y) d y,
$$

after some simple rearrangements we obtain

$$
\hat{P}_{\text {or }, \tau_{\max }}(s, L)=\frac{1}{s} \frac{\frac{\partial \hat{G}(s, L)}{\partial L}}{[1+\hat{G}(s, L)]^{2}},
$$

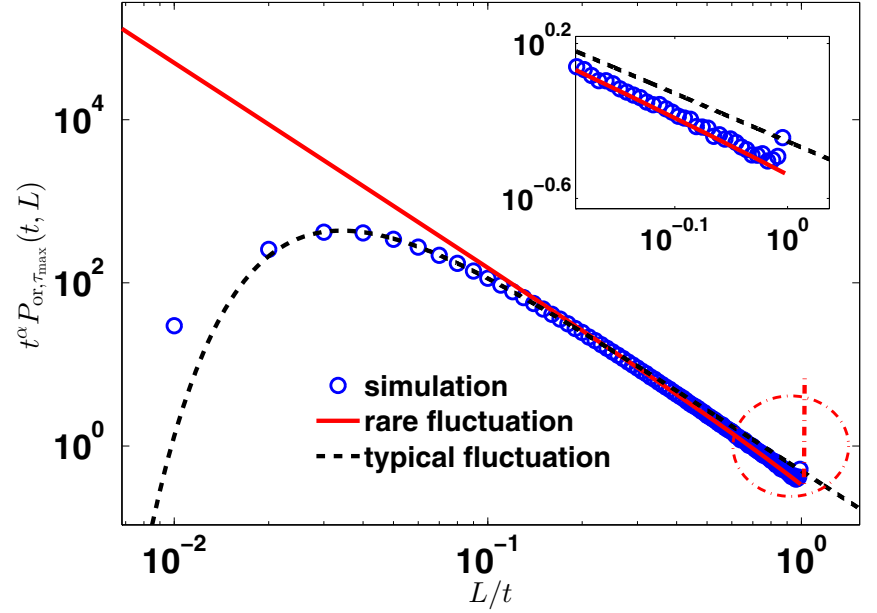

FIG. 6. Scaled PDF of the longest time interval $t^{\alpha} P_{\mathrm{or}, \tau_{\max }}(t, L)$ versus $L / t$. The red solid lines predicated by Eq. (26), or equivalently Eq. (27), describe the rare fluctuations showing the behavior of $L$ when it is of the order of $t$. The simulations, plotted by the symbols, are generated by averaging $10^{6}$ trajectories with $\alpha=3 / 2$ and $\tau_{0}=$ 1 . The figure clearly shows perfect agreement of the simulations compared with the theoretical result (26), which has a sharp cutoff at the tail of density at $\tau_{\max } / t \rightarrow 1$ (see the red dash-dotted lines). This is very different if compared with typical fluctuations calculated with Fréchet's distribution (15), which clearly does not describe well the far tail (see the inset).

where we used the relation that $P_{\text {or }, \tau_{\max }}(t, L)$ is the derivative of Eq. (22) with respect to L. Combining Eqs. (21) and (23), we have

$$
\hat{P}_{\text {or }, \tau_{\max }}(s, L) \sim \frac{1}{\hat{G}(s, L)}+\frac{\alpha}{s L \hat{G}(s, L)}+\cdots .
$$

Note that the first two terms on the right-hand side of Eq. (24), namely, $1 / \hat{G}(s, L)$ and $\alpha /(\hat{G}(s, L) s L)$, are comparable when $s L \rightarrow$ const. Hence, from Eqs. (19) and (24) we get

$$
\hat{P}_{\mathrm{or}, \tau_{\max }}(s, L) \sim \frac{\left(\tau_{0}\right)^{\alpha}}{\langle\tau\rangle} \frac{\exp (-s L)}{s L^{\alpha}}\left(1+\frac{\alpha}{s L}\right) .
$$

Taking the inverse Laplace transform $s \rightarrow t$ of Eq. (25) gives our second main result with the scaling $L \propto t$,

$$
P_{\mathrm{or}, \tau_{\max }}(t, L) \sim \frac{\left(\tau_{0}\right)^{\alpha}}{t^{\alpha}\langle\tau\rangle}\left[\alpha\left(\frac{L}{t}\right)^{-\alpha-1}-(\alpha-1)\left(\frac{L}{t}\right)^{-\alpha}\right],
$$

with $0 \leqslant L \leqslant t$. The theoretical predication of Eq. (26) is compared with numerical simulations in Fig. 6. As explained before, Eq. (26) describing the far tail of the distribution of $\tau_{\text {max }}$ is a modification of Fréchet's law.

According to Eq. (26), we find

$$
\lim _{t \rightarrow \infty}\langle\tau\rangle\left(\frac{t}{\tau_{0}}\right)^{\alpha} P_{\mathrm{or}, \tau_{\max }}(t, L)=\mathcal{I}_{\mathrm{or}, \alpha}\left(\frac{L}{t}\right),
$$

where

$$
\mathcal{I}_{\text {or }, \alpha}(y)=\alpha y^{-\alpha-1}-(\alpha-1) y^{-\alpha},
$$

with $0<y<1$. This scaling solution describes the far tail of the distribution, where Fréchet's law does not work. In fact, 
these two laws are related as the $y^{-\alpha-1}$ term matches the far tail of the Fréchet law, as it should. Since $0<y<1$ implies $\tau_{\max }<t$, moments of $\tau_{\max }$ are computed with respect to this scaling solution. In contrast, the Fréchet law gives diverging variance of $\tau_{\max }$, which is certainly not a possibility since $\tau_{\max }$ is bounded. The expression in Eq. (27) is an infinite density describing a non-normalizing limiting law. More exactly, $\mathcal{I}_{\text {or }, \alpha}(\cdot)$ is not normalizable; the moments of order $q>\alpha$ of $\tau_{\max }$ are calculated with respect to this non-normalized state. (For more details on infinite densities see Refs. [46-50].)

\section{B. Rare fluctuations of the position}

We now investigate the distribution of $x$ proving the validity of the big jump principle (9). Let $P_{\text {or }}(x, t)$ be the PDF of finding the walker on $x$ at time $t$. The starting point is the well-known Montroll-Weiss equation, which gives the Fourier-Laplace transform of $P_{\mathrm{or}}(x, t)[10,17]$,

$$
\tilde{\hat{P}}_{\text {or }}(k, s)=\frac{1-\hat{\phi}(s)}{s} \frac{1}{1-\tilde{f}(k) \hat{\phi}(s)},
$$

with $\tilde{\hat{P}}_{\text {or }}(k, s)=\int_{-\infty}^{\infty} \int_{0}^{\infty} \exp (i k x-s t) P_{\text {or }}(x, t) d t d x$. Here $\tilde{f}(k)$ is the Fourier transform of the jump length PDF $f(\chi)$ and $\hat{\phi}(s)$ is the Laplace transform of waiting-time PDF. The long-wavelength approximation, i.e., the small- $s$ and $-k$ limit, is routinely applied to investigate the long-time limit of $P_{\text {or }}(x, t)$. However, how to choose the limit of $k \rightarrow 0$ and $s \rightarrow 0$ is actually slightly subtle. Utilizing Eqs. (3) and (5) and assuming that the ratio $\left|s^{\alpha}\right| /|k|$ is fixed, we get

$$
\tilde{\hat{P}}_{\text {or }}(k, s) \sim \frac{\langle\tau\rangle}{-i k a+s\langle\tau\rangle-\left(\tau_{0}\right)^{\alpha}|\Gamma(1-\alpha)| s^{\alpha}} .
$$

Inverting, we then find a known limit theorem [51,52]

$$
P_{\mathrm{or}}(x, t) \sim \frac{1}{a(t / \bar{t})^{1 / \alpha}} L_{\alpha, 1}\left(\frac{x-a t /\langle\tau\rangle}{a(t / \bar{t})^{1 / \alpha}}\right),
$$

where $\bar{t}=\langle\tau\rangle^{1+\alpha} /\left(\tau_{0}\right)^{\alpha}|\Gamma(1-\alpha)|, L_{\alpha, 1}(\cdot)$ is the nonsymmetrical Lévy stable law with characteristic function $\exp \left[(i k)^{\alpha}\right]$, and $a>0$. This central limit theorem, just like Fréchet's law, has its limitations. As a stand alone formula, it predicates $\left\langle x^{2}(t)\right\rangle=\infty$, since the second moment of the Lévy distribution does not exist. This means that we must consider a different method to describe the far tail.

To proceed we reanalyze Eq. (29) but now fixing $|s| /|k|$. This is a large-deviation approach since such a scaling implies a ballistic scaling behavior of $x$ and $t$, unlike $x-a t /\langle\tau\rangle \propto$ $t^{1 / \alpha}$ in Eq. (31). The strategy we use now, i.e., the determination of $P_{\text {or }}(x, t)$ for $x \propto t$, is similar to the approach in the preceding section where we calculated $P_{\text {or }, \tau_{\max }}(t, L)$. The obvious difference is that there we started with Eq. (16), while here we start with the Montroll-Weiss equation (29). More specifically, in Sec. III A we assumed that $s L \propto$ const, while here $|s|$ and $|k|$ are small and of the same order, where $s$ and $k$ are a Laplace pair and a Fourier pair of $t$ and $x$, respectively.
We restart from Eq. (29), which gives

$$
\tilde{\hat{P}}_{\text {or }}(k, s) \sim \underbrace{\frac{\langle\tau\rangle}{\langle\tau\rangle s-i k a}}_{\text {leading }}+\underbrace{\frac{i k a\left(\tau_{0}\right)^{\alpha}|\Gamma(1-\alpha)| s^{\alpha-1}}{(s\langle\tau\rangle-i k a)^{2}}}_{\text {correction }}+\cdots .
$$

The derivation of Eq. (32) is given in Appendix A. The inversion of the leading term is trivial, but it yields a $\delta$ function $\delta(x-a t /\langle\tau\rangle)$. Mathematically we choose a scaling that shrinks the density to an uninteresting object. Luckily, the correction term is important as it describes the far tail. So for $x \neq a t /\langle\tau\rangle$ we have

$$
P_{\text {or }}(x, t) \sim \mathcal{F}_{k \rightarrow x}^{-1} \mathcal{L}_{s \rightarrow t}^{-1}\left[\frac{a\left(\tau_{0}\right)^{\alpha}|\Gamma(1-\alpha)| i k s^{\alpha-1}}{(s\langle\tau\rangle-i k a)^{2}}\right],
$$

with $\mathcal{F}_{k \rightarrow x}^{-1}$ and $\mathcal{L}_{s \rightarrow t}^{-1}$ the inverse Fourier and inverse Laplace transforms, respectively. We first perform the inverse Laplace transform using the convolution theorem and the pairs

$$
\begin{aligned}
\mathcal{L}_{s \rightarrow t}^{-1}\left[s^{\alpha-1}\right] & =\frac{t^{-\alpha}}{\Gamma(1-\alpha)}, \\
\mathcal{L}_{s \rightarrow t}^{-1}\left[\frac{1}{(s-i k a /\langle\tau\rangle)^{2}}\right] & =t \exp \left(i k a \frac{t}{\langle\tau\rangle}\right)
\end{aligned}
$$

and find

$$
P_{\mathrm{or}}(x, t) \sim \mathcal{F}_{k \rightarrow x}^{-1}\left[-i k \frac{a\left(\tau_{0}\right)^{\alpha}}{\langle\tau\rangle^{2}} \int_{0}^{t} \frac{y \exp \left(\frac{i k a y}{\langle\tau\rangle}\right)}{(t-y)^{\alpha}} d y\right]
$$

The inverse Fourier transform of $\exp (i k a y /\langle\tau\rangle)$ is a $\delta$ function and the $i k$ in front of this expression is the spatial derivative in $x$ space; hence we get

$$
P_{\text {or }}(x, t) \sim \frac{\left(\tau_{0}\right)^{\alpha}}{\langle\tau\rangle} \frac{\partial}{\partial x} \int_{0}^{t} \frac{y \delta\left(y-\frac{x\langle\tau\rangle}{a}\right)}{(t-y)^{\alpha}} d y .
$$

Then, after simple rearrangement,

$$
P_{\text {or }}(x, t) \sim \frac{\left(\tau_{0}\right)^{\alpha}}{a t^{\alpha}} \mathcal{I}_{\text {or }, \alpha}(\xi),
$$

with $0<\xi<1, \xi=1-(x / a) /(t /\langle\tau\rangle)$, and $\mathcal{I}_{\text {or }, \alpha}(\cdot)$ defined by Eq. (28). As Fig. 7 demonstrates, this equation describes the far tail of the density of the spreading packet and it is complementary to the Lévy law (31). The MSD of the process is obtained with respect to integration over the formula (37) and in that sense this equation resolves the drawback of the Lévy density. More important is the fact that the distributions of $\tau_{\max }$ [Eq. (27)] and $x$ [Eq. (37)] have the same structure, beyond a trivial Jacobian. In other words, given the fact that these observables have the same distribution, we have proven the single big jump principle (9) for the ordinary processes. The statistics of one waiting time $\tau_{\max }$ determines the fluctuations at small $x$. In addition, since Eq. (37) gives the MSD, which is used in most experimental, theoretical, and numerical works to characterize the fluctuations, we see that the MSD is directly related to the single big jump principle and extreme value statistics. One should note that low-order moments like $\left\langle|x-\langle x\rangle|^{q}\right\rangle$, with $q<\alpha$, are finite with respect to the Lévy density and these are given by integration with respect to Eq. (31). 


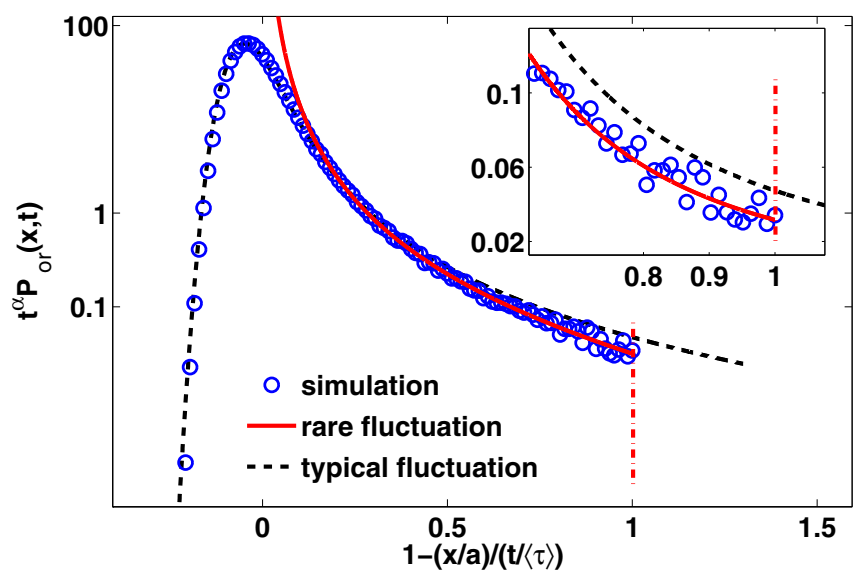

FIG. 7. Scaled PDF $P_{\text {or }}(x, t)$ compared with the prediction of the single big jump principle and the Lévy central limit theorem describing rare and typical fluctuations. The parameters are $a=$ $1, \sigma=1, \alpha=1.5$, and $\tau_{0}=0.1$, and for the simulation we used $5 \times 10^{6}$ trajectories. The inset exhibits a comparison among typical fluctuations (31), rare fluctuations (37), and simulations. Clearly, our theory performs perfectly, while Eq. (31) overshoots (see the inset) and extends to positive infinity. In reality there is a clear cutoff at $x=0$ being exclusively revealed by the single big jump principle analysis (see the red dash-dotted lines).

Remark 2. We now study the case of the CTRW in two dimensions and focus on an ordinary process. The joint length PDF is $f\left(\chi_{x}, \chi_{y}\right)=f_{x}\left(\chi_{x}\right) f_{y}\left(\chi_{y}\right)$, where $f_{x}\left(\chi_{x}\right)$ is the same as in Eq. (2) and $f_{y}\left(\chi_{y}\right)=\exp \left[-\left(\chi_{y}\right)^{2} / 2\left(\sigma_{y}\right)^{2}\right] / \sqrt{2 \pi\left(\sigma_{y}\right)^{2}}$, with $\sigma_{y}$ a constant. This means that the drift is only in the $x$ direction. Similar to our previous calculations, we use the Montroll-Weiss equation to find

$$
P_{\mathrm{or}}(x, y, t) \sim \frac{\left(\tau_{0}\right)^{\alpha}}{a t^{\alpha}} \mathcal{I}_{\mathrm{or}, \alpha}(\xi) \delta(y) .
$$

The marginal density $P_{\mathrm{or}}(x, t)$ is the same as the onedimensional case (37). Note that $\tau_{\max }$ is of the order $t$ (for the far tail), so in the $y$ direction the particles are practically frozen. Hence we get a $\delta$ function since there is no drift in the $y$ direction.

\section{EQUILIBRIUM CASE}

Up to now we have considered the case when a physical clock was started immediately at the beginning of the process, i.e., an ordinary CTRW. Here we consider the equilibrium initial condition. We note that for $0<\alpha<1$, i.e., when the average trapping time diverges, this is related to the aging CTRW [33,34,40,53,54], which is used as a tool to describe complex systems ranging from the Anderson insulator to colloidal suspensions and was first introduced by Monthus and Bouchaud to illustrate the diffusion in glasses [55]. In contrast, when $1<\alpha<2$ and Eq. (1) holds, we have a stationary process. Then, as mentioned already, the mean waiting time for the first event is infinite [see Eq. (7)]. In practice, if we start the process at time $-t_{a}$ and $t_{a}$ is large but finite, the averaged first waiting time observed after time $t_{a}$ will increase with $t_{a}$, and when $t_{a}$ tends to infinity it will diverge. Here we focus on the statistics of particles with an equilibrium condition, i.e., $t_{a} \rightarrow \infty$.

\section{A. Rare fluctuations of the position}

In Fourier-Laplace space, the density of spreading particles is given by [33]

$$
\tilde{\hat{P}}_{\mathrm{eq}}(k, s)=\frac{1-\hat{h}_{\mathrm{eq}}(s)}{s}+\frac{[1-\hat{\phi}(s)] \hat{h}_{\mathrm{eq}}(s) \tilde{f}(k)}{s[1-\hat{\phi}(s) \tilde{f}(k)]} .
$$

This equation is a modification of the Montroll-Weiss equation, taking into consideration the equilibrium initial state. Using the Laplace transform of Eq. (1), we have

$$
\tilde{\hat{P}}_{\mathrm{eq}}(k, s)=\frac{\langle\tau\rangle s-1+\tilde{\phi}(s)}{\langle\tau\rangle s^{2}}+\frac{[1-\hat{\phi}(s)]^{2} \tilde{f}(k)}{\langle\tau\rangle s^{2}[1-\hat{\phi}(s) \tilde{f}(k)]} .
$$

The first term on the right-hand side is $k$ independent; hence its inverse Fourier transform gives a $\delta$ function on the initial condition $x=0$ describing nonmoving particles. This population of motionless particles is non-negligible in the sense that they contribute to the MSD [see Eq. (B6)].

Based on Eq. (40), we consider typical fluctuations, i.e., $k, s \rightarrow 0$ and $|k| \propto\left|s^{\alpha}\right|$,

$$
\begin{aligned}
\tilde{\hat{P}}_{\mathrm{eq}}(k, s) & \sim \frac{[1-\hat{\phi}(s)]^{2}}{\langle\tau\rangle s^{2}} \frac{1}{1-\hat{\phi}(s) \tilde{f}(k)} \\
& \sim \frac{\langle\tau\rangle}{\langle\tau\rangle s-i k a-b_{\alpha} s^{\alpha}},
\end{aligned}
$$

where we used the asymptotic behaviors of $\tilde{\phi}(s)$ and $\hat{f}(k)$. The inverse Laplace-Fourier transform of Eq. (41) yields

$$
P_{\mathrm{eq}}(x, t) \sim \frac{1}{a(t / \bar{t})^{1 / \alpha}} L_{\alpha, 1}\left(\frac{x-a t /\langle\tau\rangle}{a(t / \bar{t})^{1 / \alpha}}\right),
$$

According to Eq. (42), the typical fluctuations are the same as the one of the ordinary case [see Eq. (31) and the dashed lines in Fig. 8]. That is, the bulk fluctuations do not depend on the initial state. On the other hand, the MSDs of both cases are different, which means that the far tail of $P_{\mathrm{eq}}(x, t)$ should be modified compared with the ordinary case. As mentioned before, the normalized density (42) gives an unphysical infinite MSD due to the slowly decaying tail of the asymmetric Lévy distribution. This means that we expect modifications of this limiting law at the far tail.

For the rare events of the equilibrium CTRW, i.e., both $s$ and $k$ are small and comparable, inserting $\hat{\phi}(s)$ and $\tilde{f}(k)$ into Eq. (40) gives

$$
\tilde{\hat{P}}_{\mathrm{eq}}(k, s) \sim \frac{b_{\alpha}}{\langle\tau\rangle s^{2-\alpha}}+\frac{\langle\tau\rangle-2 b_{\alpha} s^{\alpha-1}}{\langle\tau\rangle s-i k a-b_{\alpha} s^{\alpha}} .
$$

Rewriting the second term on the right-hand side of Eq. (43) as

$$
\frac{\langle\tau\rangle-2 b_{\alpha} s^{\alpha-1}}{\langle\tau\rangle s-i k a-b_{\alpha} s^{\alpha}} \sim \frac{\langle\tau\rangle-b_{\alpha} s^{\alpha-1}}{\langle\tau\rangle s-i k a}+\frac{b_{\alpha} s^{\alpha-1} i k a}{(\langle\tau\rangle s-i k a)^{2}}
$$

and using the relation

$$
\mathcal{F}^{-1} \mathcal{L}^{-1}\left[\frac{s^{\alpha-1}}{\langle\tau\rangle s-i k a}\right]=\frac{\left(t-\frac{\langle\tau\rangle}{a} x\right)^{-\alpha}}{a \Gamma(1-\alpha)},
$$




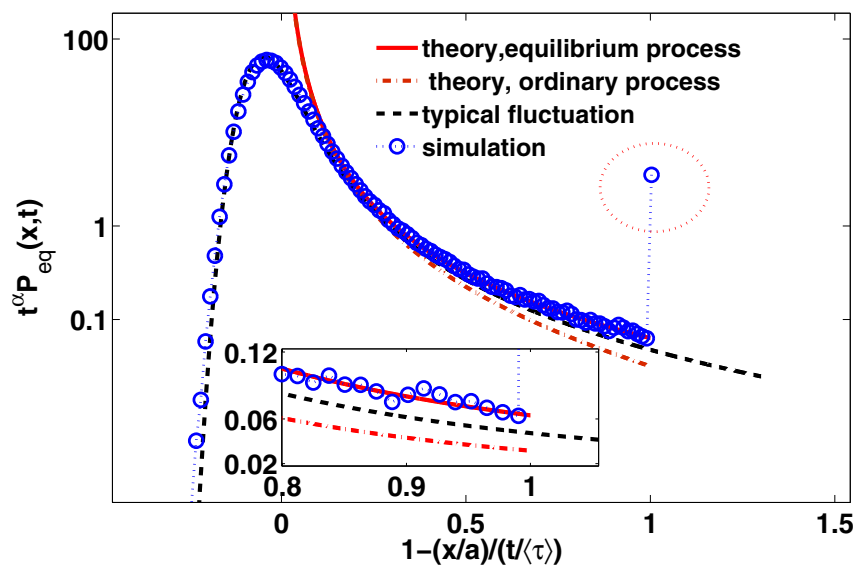

FIG. 8. Scaled PDF of the position versus $1-(x / a) /(t /\langle\tau\rangle)$. The symbols are simulation results obtained from $3 \times 10^{6}$ realizations. The red solid line calculated from Eq. (46) describing the behavior when $x \propto t$ is consistent with the far tail of the simulation results (see the inset). We also plot the theory of an ordinary process (37), showing that it clearly fails, and it underestimates the rare fluctuations described by the equilibrium theory. Notice that the $\delta$-like contribution, circled in red, describes nonmoving particles at $x=0$. Here $\alpha=1.5, a=1, \sigma=1, t_{a}=10^{4}, t=1000$, and $\tau_{0}=$ 0.1 .

we get the main results of this section describing the packet when $x$ is of the order of $t$,

$$
P_{\mathrm{eq}}(x, t) \sim \frac{\left(\tau_{0}\right)^{\alpha} t^{1-\alpha}}{\langle\tau\rangle(\alpha-1)} \delta(x)+\frac{\left(\tau_{0}\right)^{\alpha}}{a t^{\alpha}} \mathcal{I}_{\mathrm{eq}, \alpha}(\xi),
$$

where the non-normalized state function reads

$$
\mathcal{I}_{\text {eq }, \alpha}(\xi)=\alpha \xi^{-\alpha-1}+(2-\alpha) \xi^{-\alpha}
$$

and $\xi=1-(x / a) /(t /\langle\tau\rangle)$. Comparing with Eq. (37), we see that the infinite densities for the equilibrium and nonequilibrium processes are different. This indicates that initial conditions influence the statistics at small position even when the measurement time is long $t \gg\langle\tau\rangle$. The rare fluctuations for the equilibrium case are larger if compared with the ordinary process; in particular, they include a $\delta$-function contribution (see the data marked in a red circle in Fig. 8). This means that particles not moving at all contribute to the rare events. Note that Eq. (46) can be matched to the far tail of the Lévy distribution Eq. (42), as it should.

We further check that the MSD is determined by the rare fluctuations (46) resulting in a different MSD compared with the ordinary process. Using the random variable $\eta=(x-$ at $/\langle\tau\rangle) /(a t /\langle\tau\rangle)$ with $-1<\eta<0$, from Eq. (46) we get

$$
\left\langle\eta^{2}\right\rangle_{\mathrm{eq}} \sim \frac{2 b_{\alpha} t^{1-\alpha}}{\langle\tau\rangle \Gamma(4-\alpha)}
$$

(see Appendix C). Similarly, $\left\langle\eta^{2}\right\rangle_{\text {or }}$ is also obtained according to Eq. (37). Utilizing Eqs. (29) and (48),

$$
\left\langle x^{2}\right\rangle-\langle x\rangle^{2} \sim \begin{cases}\frac{2 a^{2} b_{\alpha}(\alpha-1) t^{3-\alpha}}{\langle\tau\rangle^{3} \Gamma(4-\alpha)} & \text { ordinary } \\ \frac{2 a^{2} b_{\alpha} t^{3-\alpha}}{\langle\tau\rangle^{3} \Gamma(4-\alpha)} & \text { equilibrium. }\end{cases}
$$

Though the MSDs for both cases grow as a power law $t^{3-\alpha}$, the MSD for the equilibrium case is larger than the ordinary one. Since the mean of the first waiting time following Eq. (1) is infinite, the probability of particles experiencing a long trapping time increases rapidly compared with an ordinary situation. In turn, this yields considerably inactive particles which are trapped on the origin for the whole observation time $t$ lagging far behind the mean. Hence, the MSD for the equilibrium process has a deep relationship to the motionless particles [see Eq. (B6)]. It is interesting to find that the MSDs for both cases are determined by the far tail of the packet described by the infinite densities. As expected, when $\alpha \rightarrow 2$, these two processes show normal diffusion with no difference, so then the initial condition is unimportant.

\section{B. Rare fluctuations of $\tau_{\max }$}

After calculating $P(x, t)$ for small $x$, the next aim is to deal with the far tail of the PDF $\tau_{\max }$ when $\tau_{\max }$ and $t$ are comparable. From Eq. (14) we have

$$
\begin{aligned}
\frac{1}{s}-\hat{F}_{\mathrm{eq}}(s, L)= & \frac{1}{s[1+\hat{G}(s, L)]} \\
& +\frac{1-\int_{0}^{L} \exp \left(-s \tau_{1}\right) h_{\mathrm{eq}}\left(\tau_{1}\right) d \tau_{1}}{s\left(1+\frac{1}{\hat{G}(s, L)}\right)} \\
& -\int_{0}^{L} \exp \left(-s B_{t}\right) \int_{B_{t}}^{\infty} h_{\mathrm{eq}}(\tau) d \tau d B_{t},
\end{aligned}
$$

where $\hat{G}(s, L)$ is defined in Eq. (17). It gives the PDF by the derivative

$$
\hat{P}_{\mathrm{eq}, \tau_{\max }}(s, L)=-\frac{\partial\left[\frac{1}{s}-\hat{F}_{\mathrm{eq}}(s, L)\right]}{\partial L} .
$$

Note that

$$
\frac{\partial}{\partial L} \frac{1-\int_{0}^{L} \exp \left(-s \tau_{1}\right) h_{\mathrm{eq}}\left(\tau_{1}\right) d \tau_{1}}{s[1+1 / \hat{G}(s, L)]} \sim \frac{\exp (-s L)}{-s} h_{\mathrm{eq}}(L)
$$

since $\hat{G}(s, L)$ is large with $L \propto 1 / s$. Using Eqs. (51) and (52), $\hat{P}_{\text {eq, } \tau_{\max }}(s, L)$ reduces to

$$
\begin{aligned}
\hat{P}_{\mathrm{eq}, \tau_{\max }}(s, L) \sim & \frac{1}{\hat{G}(s, L)}+\frac{\alpha}{s L \hat{G}(s, L)} \\
& +\exp (-s L) \int_{L}^{\infty} h_{\mathrm{eq}}(\tau) d \tau \\
& +\frac{\exp (-s L)}{s} h_{\mathrm{eq}}(L) .
\end{aligned}
$$

Note that Eq. (53) is a uniform approximation in Laplace space which is effective for numerous $L$ and large $t$. More exactly, within this approximation, we have the only condition that the observation time $t$ is large enough without considering the scaling between $t$ and $L$. For the typical fluctuations, the leading term of Eq. (53) is the same as the ordinary process. Thus

$$
\int_{0}^{L} P_{\mathrm{eq}, \tau_{\max }}(t, y) d y \sim \exp \left[-\frac{t}{\langle\tau\rangle}\left(\frac{\tau_{0}}{L}\right)^{\alpha}\right] .
$$

We see that the typical fluctuations of the longest time interval of both the equilibrium and the ordinary renewal processes are 


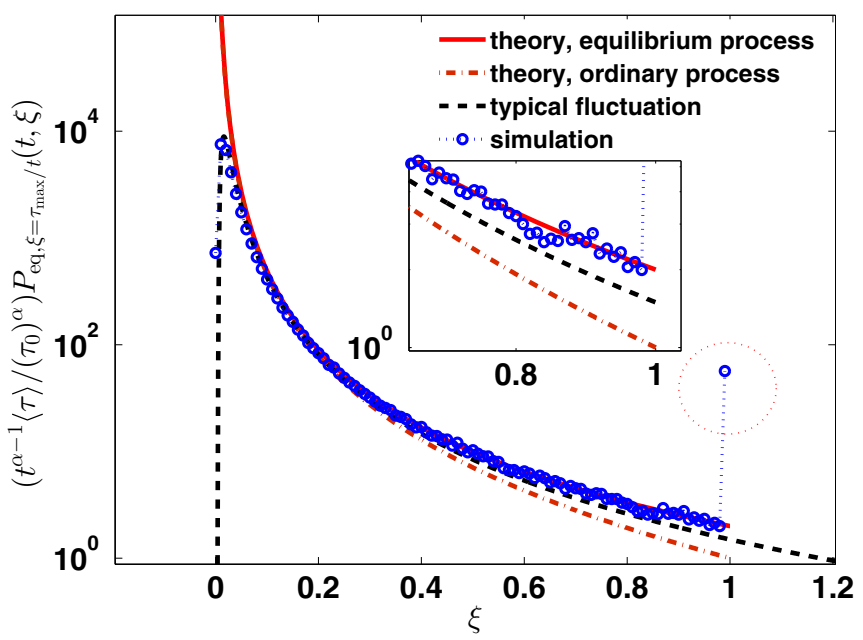

FIG. 9. Simulations of the distribution of $\xi$ with the scaling $\xi=$ $\tau_{\max } / t$ and $\alpha=1.5$ compared to the analytical predication obtained from Eq. (56). Clearly, both the typical fluctuations (54) and the rare events (37) with a nonequilibrium condition do not work at the far tail of the distribution (see the inset).

the same and independent of the initial conditions, describing the behavior when $L^{\alpha}$ is of the order of $t$.

Next we turn our attention to the case when $L \propto t$. Restarting from Eq. (53), the inverse Laplace transform gives our main result describing the far tail of the density

$$
\begin{aligned}
P_{\mathrm{eq}, \tau_{\max }}(t, L) \sim & \frac{\left(\tau_{0}\right)^{\alpha}}{t^{\alpha}\langle\tau\rangle} \mathcal{I}_{\mathrm{eq}, \alpha}(y)+\delta(t-L) \\
& \times \int_{L}^{\infty} h_{\mathrm{eq}}(\tau) d \tau+\theta(t-L) h_{\mathrm{eq}}(L),
\end{aligned}
$$

with $L \leqslant t$. Utilizing Eqs. (7) and (55), we have

$$
P_{\text {eq, } \tau_{\max }}(t, L) \sim \frac{\left(\tau_{0}\right)^{\alpha}}{t^{\alpha}\langle\tau\rangle} \mathcal{I}_{\text {eq }, \alpha}\left(\frac{L}{t}\right)+\delta(t-L) \frac{\left(\tau_{0}\right)^{\alpha} L^{1-\alpha}}{(\alpha-1)\langle\tau\rangle}
$$

(see Fig. 9). From Eqs. (46) and (56) it can be seen that the principle (9) is also valid for the equilibrium case. Though the typical fluctuations of $\tau_{\max }$ for the equilibrium and ordinary processes show no difference, their far tails are distinct from each other [see Eqs. (27) and (56)].

\section{DISCUSSION AND SUMMARY}

We have related the theory of extreme value statistics and the fluctuations of a particle diffusing in a disordered system with traps. As mentioned, the observation of a nonGaussian packet $P(x, t)$ and superdiffusive MSD has been widely reported [1-15]. Here we showed that a modification of Fréchet's law is required to fully characterize these fluctuations. The largest waiting time $\tau_{\max }$ is clearly shorter than the observation time $t$, namely, the sum $\sum_{i=1}^{N} \tau_{i}+B_{t}$ is constrained; hence we naturally have deviations from the Fréchet law. In other words, the theory of independent and identically distributed random variables completely fails to describe the phenomenon of the far tail of the packet. More profound is the observation that the statistics of $\tau_{\max }$ determine the far tail of $P(x, t)$ for the ordinary and equilibrium processes. One trapping event, the longest of the lot, controls the statistics of large deviations, and this is very different if compared with standard large-deviation theory [22], where many small jumps in the same direction control the statistics.

Our work is related to the so-called single big jump principle, which was originally formulated for $N$ independent and identically distributed random variables $\left\{\vartheta_{1}, \vartheta_{2}, \ldots, \vartheta_{N}\right\}$ [28]. It states that $\sum_{i=1}^{N} \vartheta_{i} \doteqdot$ $\max \left\{\vartheta_{1}, \vartheta_{2}, \ldots, \vartheta_{N}\right\}$ when the distribution of $\vartheta_{i}$ is subexponential and for a large maximum. Note that in the CTRW model considered in this paper we do not have any large spatial jump; instead we have long sticking events where the particles do not move. More importantly, in our case the waiting times are constrained by the total measurement time, and hence correlated, and their number $N$ fluctuates. Hence the situation encountered here is simply different (though related) to the original one. Thus one aspect of our work was to modify the principle as we did in Eq. (9) and then describe the rare events with Eqs. (27), (37), (46), and (56). This allowed us to connect the big jump theory to infinite densities. The solutions describing the far tails of the distributions of $x$ and $\tau_{\max }$ are non-normalizable; still, with proper scaling, they are the limits of the perfectly normalized probability densities. For example, in Eq. (27) we multiply the normalized density $P_{\mathrm{or}, \tau_{\max }}(t, L)$ by $\langle\tau\rangle\left(t / \tau_{0}\right)^{\alpha}$ and then get the infinite density $\mathcal{I}_{\text {or }, \alpha}(L / t)$. The variance of $\tau_{\max }$ and the superdiffusive MSD are calculated with these non-normalized states, meaning that these quantifiers of the anomaly are sensitive to rare events.

We showed that the initial condition is an important factor controlling the behavior of the far tail of distribution of interest. We calculated these for the stationary and ordinary renewal processes, showing that for the stationary process motionless particles give an important contribution to the description of the rare fluctuations and the MSD. This implies that the far tails are nonuniversal in their shapes. This can therefore be used to characterize the nature of the underlying process. As for universality, this shows up in the principle of big jump (9), as the relation between the trapping time and the position $x$ is independent of the underlying process.

We note that the surprising superdiffusion of a biased tracer in a crowded medium was also found based on a many-body theory $[5,8,56]$ and diffusion of contamination in disordered systems, as well as for numerical simulations of glass-forming systems [4,6], where it is interesting to check the relation of the dynamics and the big jump principle. The investigation of the single big jump principle in the context of other models of random walks in random environments is of great interest. For example, the biased quenched trap model exhibits typical fluctuations which are the same as those found for the biased CTRW $[9,10,57,58]$. Whether this will repeat for the rare events is still unknown. Recently, the case of $N$ independent and identically distributed random variables constrained to have a given sum was investigated, and under certain conditions the Fréchet law was found [59-61]. From this constraint it is clear that the far tail of the distribution of the maximum cannot be modeled with the Fréchet law since there is a cutoff at the far tail. It would be of interest to investigate the far tail of this model (there $N$ was fixed, while here $N$ is random) and see if the non-normalized density is found here as well. 


\section{ACKNOWLEDGMENTS}

The authors would like to thank the anonymous reviewers for their constructive comments. E.B. acknowledges the Israel Science Foundation for support through Grant No. 1898/17. W.W. was supported by a Sandwich fellowship.

\section{APPENDIX A: CALCULATION OF EQ. (32)}

We now present a detailed derivation of Eq. (32), starting from the Montroll-Weiss equation (29). Here we are interested in the case $x-a t /\langle\tau\rangle \propto a t /\langle\tau\rangle$ instead of $x-a t /\langle\tau\rangle \propto a t^{1 / \alpha}$ describing the typical fluctuations (discussed in the main text). In Fourier-Laplace space, this corresponds to $|s| \propto|k|$. Plugging Eqs. (3) and (5) into Eq. (32) leads to

$$
\begin{aligned}
\tilde{\hat{P}}_{\mathrm{or}}(k, s) \sim & \frac{1}{\langle\tau\rangle s-i k a-\left(\tau_{0}\right)^{\alpha}|\Gamma(1-\alpha)| s^{\alpha}} \\
& \times\left[\langle\tau\rangle-\left(\tau_{0}\right)^{\alpha}|\Gamma(1-\alpha)| s^{\alpha-1}\right] \\
= & \frac{1}{(\langle\tau\rangle s-i k a)\left(1-\frac{\left(\tau_{0}\right)^{\alpha}|\Gamma(1-\alpha)| s^{\alpha}}{\langle\tau\rangle s-i k a}\right)} \\
& \times\left[\langle\tau\rangle-\left(\tau_{0}\right)^{\alpha}|\Gamma(1-\alpha)| s^{\alpha-1}\right],
\end{aligned}
$$

where we use that $\langle\tau\rangle s$ and $i k a$ are comparable and neglect the term $k^{2}$ since $k^{2} \ll|s|,|k|$. Using $1 /(1-y) \simeq 1+y$, with $y \rightarrow 0$, and $s^{2 \alpha-1} /(\langle\tau\rangle s-i k a) \propto s^{2 \alpha-2} \ll s^{\alpha-1}$, Eq. (A1) reduces to

$\tilde{\hat{P}}_{\text {or }}(k, s) \sim \frac{1}{\langle\tau\rangle s-i k a}\left(\langle\tau\rangle-b_{\alpha} s^{\alpha-1}+\frac{\left(\tau_{0}\right)^{\alpha}|\Gamma(1-\alpha)|\langle\tau\rangle}{\langle\tau\rangle s-i k a} s^{\alpha}\right)$.

Regrouping, we have

$$
\tilde{\hat{P}}_{\mathrm{or}}(k, s) \sim \frac{1}{\langle\tau\rangle s-i k a}\left(\langle\tau\rangle+\frac{i k s^{\alpha-1} a\left(\tau_{0}\right)^{\alpha}|\Gamma(1-\alpha)|}{\langle\tau\rangle s-i k a}\right),
$$

which gives Eq. (32).

\section{APPENDIX B: MOMENTS OF THE POSITION FOR THE EQUILIBRIUM CASE}

We further consider the moments of the position for an equilibrium situation by using [18]

$$
\left\langle\hat{x}^{q}(s)\right\rangle=\left.(-i)^{q} \frac{\partial^{q} \tilde{\hat{P}}_{\mathrm{eq}}(k, s)}{\partial k^{q}}\right|_{k=0}
$$

to check our theoretical result (48). For $q=1$, using Eqs. (40) and (B1), we have

$$
\langle\hat{x}(s)\rangle_{\mathrm{eq}}=\frac{a}{\langle\tau\rangle s^{2}}
$$

which yields

$$
\langle x(t)\rangle_{\mathrm{eq}}=a \frac{t}{\langle\tau\rangle} .
$$

This is the exact result growing linearly with time $t$ [see also Eq. (6)]. Note that for an ordinary process, the asymptotic behavior of $\langle x(t)\rangle$ is at $/\langle\tau\rangle$. When $q=2$, from Eq. (B1) the second moment of $x(t)$ is

$$
\left\langle x^{2}(t)\right\rangle_{\mathrm{eq}} \sim \frac{a^{2} t^{2}}{\langle\tau\rangle^{2}}+\frac{2 a^{2} b_{\alpha} t^{3-\alpha}}{\langle\tau\rangle^{3} \Gamma(4-\alpha)} .
$$

Utilizing Eqs. (B3) and (B4), the MSD is

$$
\begin{aligned}
\left\langle[x(t)-\langle x(t)\rangle]^{2}\right\rangle_{\mathrm{eq}} & =\left\langle x^{2}(t)\right\rangle_{\mathrm{eq}}-\langle x(t)\rangle_{\mathrm{eq}}^{2} \\
& \sim \frac{2 a^{2} b_{\alpha} t^{3-\alpha}}{\langle\tau\rangle^{3} \Gamma(4-\alpha)} .
\end{aligned}
$$

This demonstrates that the process shows superdiffusion, increasing faster than the ordinary process. As expected, Eq. (B5) is consistent with Eq. (49) obtained from the infinite density (46).

We further consider how motionless particles contribute to the MSD. Taking the inverse Laplace-Fourier transform on the first term on the right-hand side of Eq. (39) gives $\int_{t}^{\infty} h_{\mathrm{eq}}(\tau) d \tau \delta(x)$. From Eq. (7) one can show that

$$
\begin{aligned}
\left\langle\left(x-\langle x\rangle_{\mathrm{eq}}\right)^{2}\right\rangle_{\mathrm{eq}} & \geqslant \int_{-\infty}^{\infty}\left(x-\langle x\rangle_{\mathrm{eq}}\right)^{2} \int_{t}^{\infty} h_{\mathrm{eq}}(\tau) d \tau \delta(x) d x \\
& =\frac{a^{2}\left(\tau_{0}\right)^{\alpha} t^{3-\alpha}}{\langle\tau\rangle^{3}(\alpha-1)},
\end{aligned}
$$

where we used the relation (B3). Since the MSD grows like $t^{3-\alpha}$, clearly this term describing nonmoving particles controls the leading term of the MSD (B5), while for the nonequilibrium case we get a contribution of motionless particles to the MSD which increases as $t^{2-\alpha}$ and is negligible.

\section{APPENDIX C: CALCULATION OF MSDS USING THE INFINITE DENSITIES}

In principle, the MSDs can be calculated according to Eq. (B1). However, in the long-time limit it is easy to calculate MSDs based on the non-normalized density. This method is also valid for high-order moments [49]. From Eq. (37) the scaling behavior of $\xi=1-(x / a) /(t /\langle\tau\rangle)$ gives

$$
P_{\text {or }}(\xi, t) \sim \frac{\left(\tau_{0}\right)^{\alpha} t^{1-\alpha}}{\langle\tau\rangle} \mathcal{I}_{\text {or }, \alpha}(\xi),
$$

where $0<\xi<1$. The second moment of $\xi$ is

$$
\left\langle\xi^{2}\right\rangle_{\mathrm{or}} \sim \int_{0}^{1} \xi^{2} P_{\mathrm{or}}(\xi, t) d \xi=\frac{2\left(\tau_{0}\right)^{\alpha} t^{1-\alpha}}{\langle\tau\rangle(2-\alpha)(3-\alpha)}
$$

and $\langle x(t)\rangle \sim a t /\langle\tau\rangle$. Using the relation $\left\langle\xi^{2}\right\rangle_{\text {or }}=\langle(1-$ $\left.\left.\frac{x / a}{t /\langle\tau\rangle}\right)^{2}\right\rangle_{\text {or }}$, we have

$$
\begin{aligned}
\left\langle\left(x-\frac{a t}{\langle\tau\rangle}\right)^{2}\right\rangle_{\text {or }} & =\left\langle\left(a \xi \frac{t}{\langle\tau\rangle}\right)^{2}\right\rangle_{\text {or }} \\
& =\left(a \frac{t}{\langle\tau\rangle}\right)^{2}\left\langle(\xi)^{2}\right\rangle_{\text {or }} \\
& \sim \frac{2 a^{2}\left(\tau_{0}\right)^{\alpha} t^{3-\alpha}}{\langle\tau\rangle^{3}(2-\alpha)(3-\alpha)} \\
& =\frac{2 a^{2} b_{\alpha}(\alpha-1) t^{3-\alpha}}{\langle\tau\rangle^{3} \Gamma(4-\alpha)} .
\end{aligned}
$$


This means that the MSD of the nonequilibrium case is determined by the far tail of the density, i.e., the infinite density. Similarly, the MSD of an equilibrium process follows

$$
\left\langle\left(x-\frac{a t}{\langle\tau\rangle}\right)^{2}\right\rangle_{\mathrm{eq}} \sim \int_{-\infty}^{\infty}\left(x-\frac{a t}{\langle\tau\rangle}\right)^{2} \frac{\left(\tau_{0}\right)^{\alpha} t^{1-\alpha}}{\langle\tau\rangle(\alpha-1)} \delta(x) d x+\left(a \frac{t}{\langle\tau\rangle}\right)^{2}\left\langle\xi^{2}\right\rangle_{\mathrm{eq}} \sim \frac{2 a^{2} b_{\alpha} t^{3-\alpha}}{\langle\tau\rangle^{3} \Gamma(4-\alpha)},
$$

with $\left\langle\xi^{2}\right\rangle_{\text {eq }}=\int_{0}^{1} \xi^{2} \frac{\left(\tau_{0}\right)^{\alpha}}{t^{\alpha-1}\langle\tau\rangle} \mathcal{I}_{\text {eq }, \alpha}(\xi) d \xi$. Here we want to stress that in the case of integrable observables, one can use the nonnormalized state described by the infinite density.

[1] M. F. Shlesinger, J. Stat. Phys. 10, 421 (1974).

[2] G. Gradenigo, E. Bertin, and G. Biroli, Phys. Rev. E 93, 060105(R) (2016).

[3] F. Lechenault, R. Candelier, O. Dauchot, J.-P. Bouchaud, and G. Biroli, Soft Matter 6, 3059 (2010).

[4] D. Winter, J. Horbach, P. Virnau, and K. Binder, Phys. Rev. Lett. 108, 028303 (2012).

[5] O. Bénichou, A. Bodrova, D. Chakraborty, P. Illien, A. Law, C. Mejía-Monasterio, G. Oshanin, and R. Voituriez, Phys. Rev. Lett. 111, 260601 (2013)

[6] C. F. E. Schroer and A. Heuer, Phys. Rev. Lett. 110, 067801 (2013).

[7] S. Leitmann and T. Franosch, Phys. Rev. Lett. 118, 018001 (2017).

[8] P. Illien, O. Bénichou, G. Oshanin, A. Sarracino, and R. Voituriez, Phys. Rev. Lett. 120, 200606 (2018).

[9] J. Bouchaud, A. Comtet, A. Georges, and P. L. Doussal, Ann. Phys. (NY) 201, 285 (1990).

[10] J.-P. Bouchaud and A. Georges, Phys. Rep. 195, 127 (1990).

[11] T. Akimoto, A. G. Cherstvy, and R. Metzler, Phys. Rev. E 98 , 022105 (2018).

[12] R. Hou, A. G. Cherstvy, R. Metzler, and T. Akimoto, Phys. Chem. Chem. Phys. 20, 20827 (2018).

[13] B. Berkowitz and H. Scher, Phys. Rev. Lett. 79, 4038 (1997).

[14] B. Berkowitz, A. Cortis, M. Dentz, and H. Scher, Rev. Geophys. 44, 1 (2006).

[15] Y. Zhang, M. M. Meerschaert, and R. M. Neupauer, Water Resour. Res. 52, 2462 (2016).

[16] A. Nissan, I. Dror, and B. Berkowitz, Water Resour. Res 53, 3760 (2017).

[17] R. Metzler and J. Klafter, Phys. Rep. 339, 1 (2000).

[18] J. Klafter and I. M. Sokolov, First Steps in Random Walks: From Tools to Applications (Oxford University Press, Oxford, 2011).

[19] R. Kutner and J. Masoliver, Eur. Phys. J. B 90, 50 (2017).

[20] E. J. Gumbel, Statistics of Extremes (Dover, Mineola, 2004).

[21] Extreme Events in Nature and Society, edited by S. Albeverio, V. Jentsch, and H. Kantz (Springer, Berlin, 2006).

[22] H. Touchette, Phys. Rep. 478, 1 (2009).

[23] K. Capała, B. Dybiec, and E. Gudowska-Nowak, arXiv:1909.00196.

[24] G. Oshanin, S. Burlatsky, M. Moreau, and B. Gaveau, Chem. Phys. 177, 803 (1993).

[25] G. Oshanin, A. Rosso, and G. Schehr, Phys. Rev. Lett. 110, 100602 (2013).

[26] G. Gradenigo and E. Bertin, Entropy 19, 517 (2017).

[27] G. Gradenigo and S. N. Majumdar, J. Stat. Mech. (2019) 053206 .
[28] V. P. Chistjakov, Theory Probab. Appl. 9, 640 (1964).

[29] T. Mikosch and O. Wintenberger, Probab. Theory Relat. Fields 156, 851 (2013).

[30] D. Buraczewski, E. Damek, T. Mikosch, and J. Zienkiewicz, Ann. Appl. Probab. 41, 2755 (2013).

[31] A. Vezzani, E. Barkai, and R. Burioni, Phys. Rev. E 100, 012108 (2019).

[32] A. Vezzani, E. Barkai, and R. Burioni, arXiv:1908.10975.

[33] E. Barkai and Y. C. Cheng, J. Chem. Phys. 118, 6167 (2003).

[34] J. H. P. Schulz, E. Barkai, and R. Metzler, Phys. Rev. X 4, 011028 (2014).

[35] E. W. Montroll and G. H. Weiss, J. Math. Phys. 6, 167 (1965).

[36] J. Haus and K. Kehr, Phys. Rep. 150, 263 (1987).

[37] W. Feller, An Introduction to Probability Theory and Its Applications, 2nd ed. (Wiley, New York, 1971), Vol. II.

[38] J. K. E. Tunaley, Phys. Rev. Lett. 33, 1037 (1974).

[39] M. Lax and H. Scher, Phys. Rev. Lett. 39, 781 (1977).

[40] R. Metzler, J.-H. Jeon, A. G. Cherstvy, and E. Barkai, Phys. Chem. Chem. Phys. 16, 24128 (2014).

[41] H. Scher and E. W. Montroll, Phys. Rev. B 12, 2455 (1975).

[42] D. Krapf, G. Campagnola, K. Nepal, and O. B. Peersen, Phys. Chem. Chem. Phys. 18, 12633 (2016).

[43] Y. Edery, S. Berg, and D. Weitz, Phys. Rev. Lett. 120, 028005 (2018).

[44] C. Godrèche and J. M. Luck, J. Stat. Phys. 104, 489 (2001).

[45] C. Godrèche, S. N. Majumdar, and G. Schehr, J. Stat. Mech. (2015) P03014.

[46] J. Aaronson, An Introduction to Infinite Ergodic Theory, Mathematical Surveys and Monographs (American Mathematical Society, Providence, 1997), Vol. 50.

[47] D. A. Kessler and E. Barkai, Phys. Rev. Lett. 105, 120602 (2010).

[48] A. Rebenshtok, S. Denisov, P. Hänggi, and E. Barkai, Phys. Rev. E 90, 062135 (2014).

[49] W. L. Wang, J. H. P. Schulz, W. H. Deng, and E. Barkai, Phys. Rev. E 98, 042139 (2018).

[50] E. Aghion, D. A. Kessler, and E. Barkai, Phys. Rev. Lett. 122, 010601 (2019).

[51] M. Kotulski, J. Stat. Phys. 81, 777 (1995).

[52] R. Burioni, G. Gradenigo, A. Sarracino, A. Vezzani, and A. Vulpiani, J. Stat. Mech. (2013) P09022.

[53] G. Schütz, H. Schindler, and T. Schmidt, Biophys. J. 73, 1073 (1997).

[54] T. Kues, R. Peters, and U. Kubitscheck, Biophys. J. 80, 2954 (2001).

[55] C. Monthus and J.-P. Bouchaud, J. Phys. A: Math. Gen. 29, 3847 (1996). 
[56] L. Luo and L.-H. Tang, Phys. Rev. E 92, 042137 (2015).

[57] C. Aslangul, M. Barthélémy, N. Pottier, and D. Saint-James, J. Stat. Phys. 65, 673 (1991).

[58] S. Burov, Phys. Rev. E 96, 050103(R) (2017).
[59] M. R. Evans and S. N. Majumdar, J. Stat. Mech. (2008) P05004.

[60] C. Godrèche, J. Stat. Mech. (2019) 063207.

[61] S. N. Majumdar, A. Pal, and G. Schehr, arXiv:1910.10667. 